médecine/sciences $1995 ; 11: 1360$

\section{COURRIER}

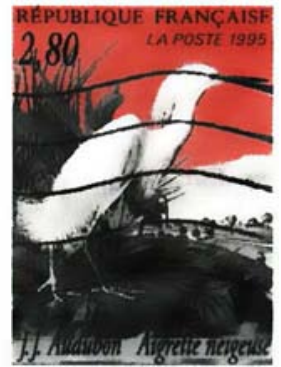

- lle exhume les hommes célèbres pour explorer leur ADN et sonder leurs mutations. Lorsqu'il s'agit d'étudier le daltonisme de John Dalton (m/s $n^{\circ} 5$, vol. 11, p. 769) par exemple, c'est fort bien : il avait lui-même souhaité que ses yeux fussent disséqués après sa mort, en ce temps où l'on espérait tout comprendre grâce aux sciences morphologiques. Nul doute qu'il se fût réjoui de connaître sa deutéranopie et de comprendre ce merveilleux mécano qu'est le gène de la vision des couleurs. Mais pour ces artistes dont l'œuvre est intimement liée à une difficulté d'être, les paris sont lâchés et l'on se demande alors où s'arrête la légitime curiosité scientifique et où commence l'effet médiatique racoleur : Vincent van Gogh avait-il une porphyrie aiguë intermittente? Frédéric Chopin était-il atteint d'une mucoviscidose ? Et surtout, tout récemment, ce souhait exprimé de

\title{
La génétique, science du futur, se penche sur le passé
}

faire exhumer Toulouse-Lautrec afin de savoir si, oui ou non, la maladie dont il était affligé était bien une pycnodysostose. Rappelons les éléments de cette controverse : en 1962, Pierre Maroteaux et Maurice Lamy individualisent une dysplasie squelettique récessive autosomique, la pycnodysostose, dont ils font une description clinique détaillée [1]. Trois ans plus tard, ils suggèrent, avec un faisceau d'arguments emportant alors la conviction de nombreux médecins, qu'Henri de Toulouse-Lautrec était atteint de ce type de nanisme. Dans un livre paru sur le peintre l'an passé [2], ce diagnostic rétrospectif est mis en doute, et son auteur, une biographe américaine, récidive [3], à l'occasion de la localisation en lq21 du gène de la pycnodysostose à partir de deux grandes familles à forte endogamie [4, 5]. Elle affirme, entre autres, que les genitalia du peintre étaient normaux sur la vue d'une mauvaise photo où, nu, de profil, sur une barque lointaine, il est à peine visible ! Pour elle, en proposant ce diagnostic posthume, les deux French dortors avaient tout simplement voulu se faire de la publicité (sels promotion).
Pour quiconque connaît Pierre Maroteaux, l'idée même est pour le moins indécente ! Aussi, de grâce, contentons-nous de contempler ses tableaux et ses caricatures et laissons le pauvre Toulouse reposer en paix.

[1. Maroteaux P, Lamy M. Presse Med $1962 ; 70$ : 999-1002.]

[2. Frey J. New York: Viking Press, 1994.]

[3. Frey J. Nature Genet $1995 ; 10$ : 128-30.]

[4. Gelb BD, et al. Nature (ienet 1995; $10: 235-7$.

[5. Polymeropoulos MH, et al. Nature (ienet 1995 ; 10 : 238-9.]

\section{Simone Gilgenkrantz}

Professeur de génétique, CHU de Nancy, laboratoire de génétique, Hôpitaux de Brabois, rue de Morvan, 54511 Vandouvre-les-Nancy Cedex, France. 